\title{
Circular RNA Expression Profile and Analysis of Their Potential Function in Psoriasis
}

\author{
Meng Qiao ${ }^{a, b} \quad$ Jian Ding ${ }^{c} \quad$ Jianjun Yan ${ }^{a} \quad$ Ronghua Lia Jian Jiao ${ }^{a} \quad$ Qing Sun $^{a}$ \\ aDepartment of Dermatology, Qilu Hospital of Shandong University, Jinan, ${ }^{b}$ Department of \\ Dermatology, Shandong Provincial Qianfoshan Hospital, Shandong University, Jinan, 'Department of \\ General Surgery, The Fourth People's Hospital of Jinan, Jinan, China
}

\section{Key Words}

Psoriasis $\bullet$ CircRNA $\bullet$ microRNA $\bullet$ miRNA response element $\bullet$ CeRNA

\begin{abstract}
Background/Aims: Circular RNAs (circRNAs) are evolutionary conserved circular non-coding RNAs that play a role in several diseases by sequestering (sponging) microRNAs (miRNAs). However, their role in psoriasis remains unclear. In the present study, we investigated the expression of circRNAs and analyzed their potential functions in psoriasis. Methods: The SBC human ceRNA array V1.0 was used to analyze circRNA expression in psoriatic lesions and normal healthy skin tissues. Functional analyses were performed using Gene ontology (GO) and Kyoto Encyclopedia of Genes and Genomes (KEGG) pathway analysis. Putative miRNA response elements (MREs) were identified using miRNA target prediction software. Six upregulated circRNAs were verified by quantitative real-time reverse transcription polymerase chain reaction in psoriatic lesions and healthy skin tissues. Results: A total of 4956 circRNAs (3016 upregulated and 1940 downregulated; fold change $\geq 2$ and $p<0.05$ ) were identified as differentially expressed in psoriasis. Furthermore, 4405 MREs were identified among the differentially expressed circRNAs. hsa_circ_0061012 was upregulated in psoriatic lesions compared with normal healthy skin tissues. The top five MREs of hsa_circ_0061012 were hsa-miR-7157-5p, hsa-miR-4769-3p, hsa-miR-6817-5p, hsa-miR-4310, and hsa-miR-6882$3 p$. GO analysis was carried out to investigate the biological functions enriched among the upregulated targets of five miRNAs in psoriasis. The GO analysis identified that most of top 30 of GO enrichment are related to psoriasis. Conclusion: hsa_circ_0061012 might be a candidate biomarker for psoriasis. The results provide a new perspective for a better understanding of ceRNA-mediated gene regulation in psoriasis, and provide a novel theoretical basis for further studies on the function of circRNA in psoriasis.




\section{Cellular Physiology Cell Physiol Biochem 2018;50:15-27 and Biochemistry Published online: 3 October $2018 \quad \begin{aligned} & \text { DOI: 10.1159/000493952 } 2018 \text { The Author(s). Published by S. Karger AG, Basel } \\ & \text { www.karger.com/cpb }\end{aligned}$ \\ Qiao et al.: Circular RNA Expression Profile in Psoriasis}

\section{Introduction}

Psoriasis is a chronic, recurrent, and immune-mediated inflammatory disease, characterized by abnormal proliferation and keratinization of keratinocytes (KCs), which is mainly mediated by $\mathrm{T}$ cells in a polygenic background [1]. The estimated global prevalence of diagnosed psoriasis varies from 2 to $4 \%$ [2], and is higher in adults (0.91 to 8.5\%) compared with that in children (0 to 2.1\%) [3]. Psoriasis has shown a marked upward trend in recent years. The course of psoriasis is comparatively long and recurrence happens frequently, which seriously affects patients' quality of life, causing great harm to their physical and mental health [4]. To date, there is no cure for psoriasis, mainly because its exact etiology and pathogenesis have not yet been fully elucidated. Many factors are involved, such as immunity, inflammation, cell proliferation, and apoptosis, and neurotransmitter and intracellular signal transduction pathways [5]. The immune factor-mediated inflammatory reaction can promote abnormal proliferation of KCs, ultimately leading to psoriasis; however, a satisfactory therapeutic effect on psoriasis can be achieved by inhibiting inflammation [6].

Since the discovery of non-coding RNAs (ncRNAs), studies have found that many ncRNAs, such as microRNAs (miRNAs) and long non-coding RNAs (lncRNAs) long are involved in the pathogenesis and development of the molecular pathology of psoriasis [7, 8]. However, the expression profile of another class of ncRNAs, circular RNAs (circRNAs), and their potential biological functions in psoriasis, remain unclear. CircRNAs, generated by alternative splicing, represent a special class of ncRNA molecules $[9,10]$. CircRNAs are abundant in the eukaryotic cell cytoplasm and show tissue, behavior, and disease specificity [11]. In contrast to linear RNAs, circRNAs are characterized by a covalently closed loop structure with neither 5' to 3' polarity, nor a polyadenylated tail. CircRNAs are resistant to RNA exonuclease, which improves their stability [12]. CircRNAs can regulate transcription or splicing, and interact with RNA binding proteins (RBPs) $[13,14]$. Remarkably, emerging evidence indicates that some circRNAs can serve as competitive endogenous RNA (ceRNA) of microRNA (miRNA) through its miRNA response elements (MREs), which can competitively binding miRNAs to release or attenuate translational repression or degradation through sequestering miRNAs away from parental mRNA $[15,16]$. Moreover, circRNAs have been reported to play essential roles in many life processes, such as aging, insulin secretion, tissue development, atherosclerotic vascular disease risk, cardiac hypertrophy, and cancer [17]. Importantly, some circRNAs were involved in regulating immunity, inflammation, and cell proliferation in many types of cells [18-20]. Because these factors contributes to psoriasis, we predicted that circRNAs also involves in psoriasis. However, nothing is known about the expression profiles and functions of circRNAs in psoriasis.

In the present study, a circRNA microarray was used to identify differentially expressed circRNAs between psoriatic lesions and normal healthy skin tissues. The expression levels of selected differentially expressed circRNAs were further verified by quantitative realtime reverse transcription PCR (qRT-PCR). Finally, we found that hsa_circ_0061012 was significantly upregulated in psoriatic lesions, indicating that hsa_circ_0061012 might be a candidate biomarker for psoriasis.

\section{Materials and Methods}

\section{Patients and tissue samples}

In this study, thirteen psoriatic lesions and six normal healthy skin tissues were collected from the Department of Dermatology, Qilu Hospital of Shandong University. All psoriatic lesions were consistent with the diagnostic criteria for psoriasis vulgaris and scored by the psoriasis area and severity index (PASI). The normal control group comprised healthy adult volunteers, whose age, sex, and site of extraction were matched with the psoriasis group. Details of the samples are shown in Table 1. Sample weight was evaluated before the study was conducted. Three lesions, the weight of that is more than $50 \mathrm{mg}$, were used for microarray detection, and the other ten, the weight of that is less than $50 \mathrm{mg}$, were used for qRT-PCR verification. 


\section{Cellular Physiology Cell Physiol Biochem 2018;50:15-27 and Biochemistry Published online: 3 October $2018 \quad \begin{aligned} & \text { DOI: 10.1159/000493952 } 2018 \text { The Author(s). Published by S. Karger AG, Basel } \\ & \text { www.karger.com/cpb }\end{aligned}$ \\ Qiao et al.: Circular RNA Expression Profile in Psoriasis}

Psoriasis case eligibility criteria: no glucocorticoids, immunosuppressants, and biological agents were used for 3 months before specimen collection. Patients with psoriasis were not treated with ultraviolet light for 1 month before specimen collection. No glucocorticoids were applied 2 weeks before specimen collection. No congenital or acquired autoimmune diseases and other immune related diseases were present. No coagulopathy and other unsuitable surgical diseases were present. Informed consent was obtained from all individual participants included in this study. The study was approved by the ethics committee of Qilu Hospital of Shandong University. All tissue samples were preserved at $-80{ }^{\circ} \mathrm{C}$ until RNA extraction.

\section{CircRNA microarray analysis}

RNA extraction and purification. Total RNAs from three psoriatic lesions and three normal healthy skin tissues were extracted and purified using RNeasy mini kit (Cat\#74106, Qiagen, GmBH, Hilden, Germany). The RNA integrity coefficient (RIN) number, as a measure of RNA integrity, was checked using an Agilent Bioanalyzer 2100 (Agilent technologies, Santa Clara, CA, USA). The purity and quantity of initial total RNA chip samples were determined using a NanoDrop ND2000 spectrophotometer (NanoDrop, Wilmington, DE, USA) and an Agilent Bioanalyzer 2100 for quality inspection, to exclude genomic DNA (gDNA) contamination. RNA integrity was also determined by electrophoresis through a denaturing agarose gel. Qualified RNA was used in the following chip experiments.

RNA amplification and labeling. Total RNA was amplified and labeled using a Low Input Quick Amp WT Labeling Kit (Cat.\# 5190-2943, Agilent technologies). Labeled cRNAs were purified using an RNeasy mini kit (Cat. \# 74106, Qiagen).

Microarray hybridization. The SBC Human (4*180K) ceRNA array V1.0 (Shanghai Biotechnology Corporation, Shanghai, China) was used to detect circRNA and mRNA expression profiles. Each slide was hybridized with $1.65 \mu \mathrm{g}$ of Cy3-labeled cRNA using a Gene Expression Hybridization Kit (Cat.\# 51885242, Agilent technologies) in a Hybridization Oven (Cat.\# G2545A, Agilent technologies), according to the manufacturer's instructions. After $17 \mathrm{~h}$ of hybridization, the slides were washed in staining dishes (Cat.\# 121, Thermo Shandon, Waltham, MA, US) using a Gene Expression Wash Buffer Kit (Cat.\# 5188-5327, Agilent technologies).

\section{Data acquisition}

Slides were scanned by an Agilent Microarray Scanner (Cat\#G2565CA, Agilent technologies). Data were extracted using Feature Extraction software 10.7 (Agilent technologies). Quantile normalization and subsequent data processing were performed using the R software package (provided by Shanghai Biotechnology Corporation, Shanghai, China). Statistically significant differentially expressed circRNAs and mRNAs between the two groups were defined as fold change $\geq 2$ and P-value $<0.05$, as estimated by a t-test.

\section{GO and KEGG pathway analysis}

Gene Ontology (GO) (www.geneontology.org) is a widely used ontology in the bioinformatics field. We analyzed the association of the differentially expressed mRNAs with Biological Process (BP), and Molecular Function (MF) in the GO database. The P value denotes the significance of the GO term enrichment among the differentially expressed mRNAs. Kyoto Encyclopedia of Genes and Genomes (KEGG) pathway analysis is a systematic analysis of gene function and genome information database, which helps researchers to study gene and expression information as a whole network. KEGG enrichment analysis of differentially expressed genes can identify differentially enriched pathways, which is helpful to find the biological regulation pathways that were significantly altered under the experimental conditions. The enrichment analysis was performed using Fisher's exact test in clusterProfiler from R/bioconductor (https://www.bioconductor. 


\section{Cellular Physiology Cell Physiol Biochem 2018;50:15-27 and Biochemistry Published online: 3 October $2018 \quad \begin{aligned} & \text { DOI: 10.1159/000493952 } 2018 \text { The Author(s). Published by S. Karger AG, Basel } \\ & \text { www.karger.com/cpb }\end{aligned}$ \\ Qiao et al.: Circular RNA Expression Profile in Psoriasis}

org/). The standard of selection was the number of genes that fall on a GO term/ or pathway $\geq 2$, with a $P$-value $<0.05$.

\section{qRT-PCR}

QRT-PCR was performed using a 7900 HT Sequence Detection System (ABI, Foster City, CA, USA) to evaluate the expression of six upregulated circRNAs (hsa_circ_0031329, hsa_circ_002269, hsa_circ_0061012, hsa_circ_0043804, hsa_circ_0062533, and hsa_circ_0069260). Total RNA was extracted from the samples using RNeasy mini kit (Cat\#74106, Qiagen, GmBH, Hilden, Germany). CDNAs were prepared using random primers and a ReverTra Ace qPCR Kit (Toyobo, Osaka, Japan, FSQ-101). A power SYBR Green PCR Master Mix (ABI, 4368708) was used for the PCR reaction. The primer sequences of specific genes are shown in Table 2.

miRNA response elements (MREs) predication

The putative MREs of differentially expressed circRNAs and mRNAs were described bioinformatically using the Arraystar miRNA target prediction software (Arraystar, Rockville, MD, USA).

\section{miRNA target predication and network construction}

The targets of hsa-miR-7157-5p, hsa-miR-4769-3p, hsa-miR-6817-5p, hsa-miR-4310, and hsa-miR6882-3p were predicated using Targetscan (http://www.targetscan.org/vert_71/) and miRDB (http:// www.mirdb.org/). The predicated targets that were upregulated in psoriatic lesions were used to construct a miRNAs and targets network using Cytoscape (http://www.cytoscape.org/).

\section{Statistical analysis}

All data were expressed as mean \pm standard deviation (SD). The comparison between two groups were analyzed using Student's t-test using SPSS 15.0 software (SPSS, Chicago, IL, USA), a P-value $<0.05$ was considered statistically significant.

\section{Results}

Expression pattern of circRNAs and $m R N A s$

To study the expression pattern of circRNAs in psoriatic lesions and normal healthy skin tissues, we used a circRNA microarray to identify differentially expressed circRNAs. A box plot was used to evaluate the distribution of the hybridization data and the degree of dispersion. The box plot showed that after log2 normalization, no abnormal distributions of data were found in the six samples (Fig. 1A). Pearson`s correlation coefficient r was calculated for correlation analysis between psoriatic lesions and normal healthy skin tissues (Fig. 1B). The variation between psoriatic lesions and normal healthy skin tissues was displayed in a scatter plot of the circRNA expression profile (Fig. 1C). In addition, a volcano plot identified differentially expressed circRNAs at different P-values and fold-changes between the two groups (Fig. 1D). Hierarchical clustering revealed that the circRNA expression levels were distinguishable in the associated heat map (Fig. 2). The analysis identified a set of 4956 differentially expressed circRNAs (Supplementary Table S1 - for all supplementary material see www.karger.com/10.1159/000493952/). The distribution of dysregulated circRNAs on human chromosomes is shown in Fig. 3. Among all the differential expressed circRNAs, 


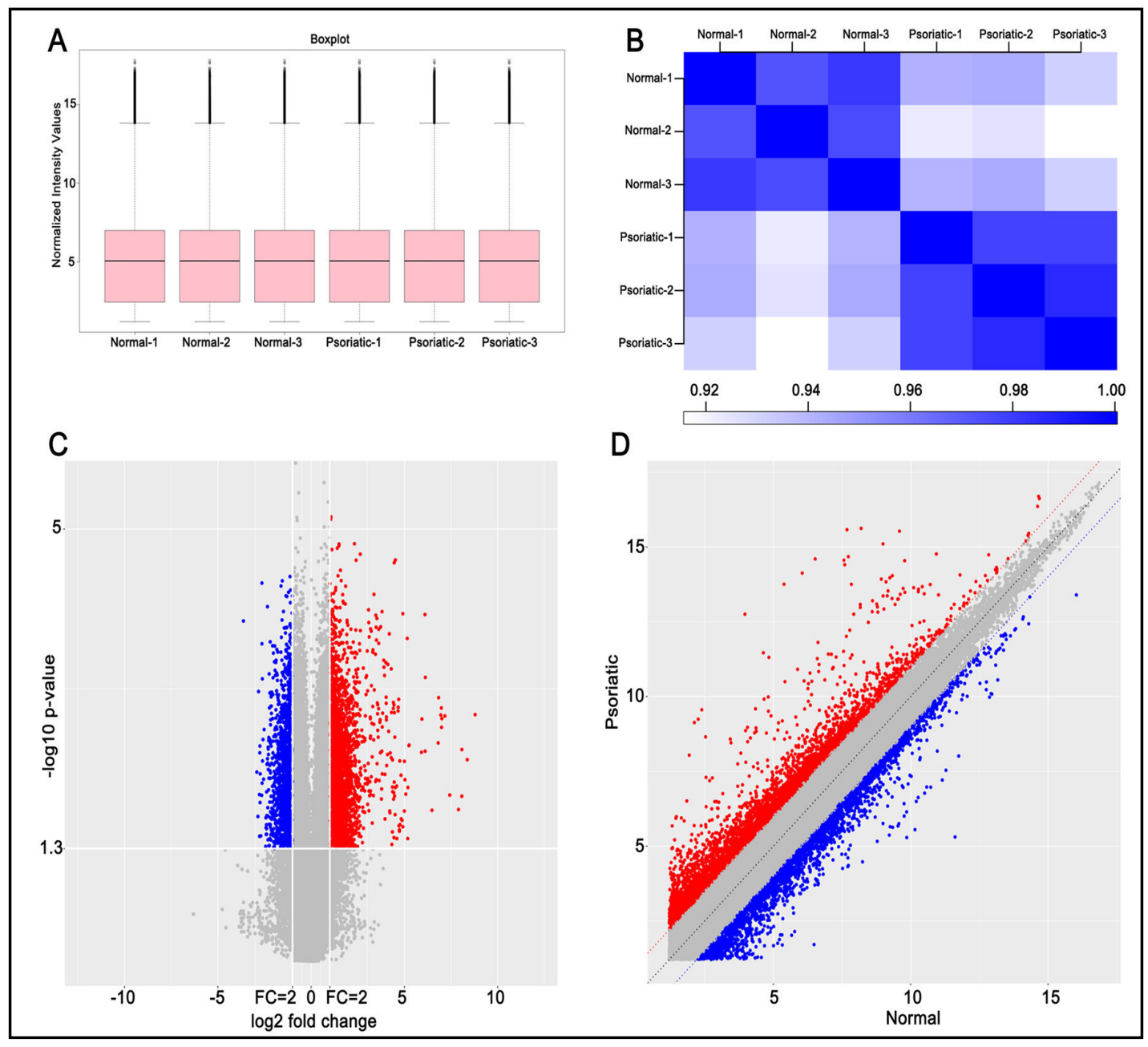

Fig. 1. Expression profile of circular RNAs (circRNAs) detected using a microarray in psoriatic lesions compared with normal healthy skin tissues. A: Box plots were applied to visualize the distributions of circRNAs for the two groups. After normalization, the distributions of the $\log 2$ ratios among six samples were nearly the same. B: Block diagram for the correlation coefficient $r$. The brightness of the color represents the degree of correlation between samples, and the deeper the brightness, the higher the correlation between the two samples. C: Volcano Plot visualizing differential circRNA expression between the two groups. The vertical lines correspond to a 2.0-fold change (FC) (log2 scaled) (upregulation and downregulation, respectively). The horizontal line represents a P-value of 0.05 ( $\log 10$ scaled). The red and blue points in the plot represent the statistically significantly upregulated and downregulated circRNAs, respectively. Red squares mark differentially expressed circRNAs in psoriatic lesions versus normal healthy skin tissues $(\mathrm{P}<0.05, \mathrm{FC} \geq 2)$. Blue squares mark differentially expressed circRNAs in in psoriatic lesions compared with normal healthy skin tissues. $(\mathrm{P}<0.05, \mathrm{FC} \leq 0.5)$. D: Scatter Plot demonstrating the variation of circRNAs expression in Psor ( $\mathrm{y}$-axis) versus Nor (x-axis). The values of the $\mathrm{x}$ - and $\mathrm{y}$-axes are the averaged normalized signal values of each group (log2 scaled). The middle green line represents no difference between the two groups. A point outside the 45-degree line in the figure. In the figure, representing the probe point in the two groups, indicates that the signal difference is $\mathrm{FC} \geq 2$; red represents upregulation and blue represents downregulation.

3016 were upregulated and 1940 were downregulated (fold change $\geq 2$ and $\mathrm{P}<0.05$ ) (Supplementary Table S 1). In the present study, the top 20 upregulated and downregulated circRNAs (based on fold change) are shown in Table 3. In addition, 1156 upregulated mRNAs and 569 downregulated mRNAs were found in psoriatic lesions compared with normal healthy skin tissues (Supplementary Table S2). 


\section{Cellular Physiology and Biochemistry

GO and KEGG pathway analysis of the differentially expressed $m R N A s$

To identify the possible etiological factors and key genes in the pathogenesis of psoriasis, we analyzed the GO enrichment of differentially expressed mRNAs and the results are shown in Supplementary Table S3. We analyzed the association of these differentially expressed mRNAs with BP and MF in the GO database. Through GO enrichment analysis, the important functions that are associated with the differences between the two groups and the corresponding genes can be found. The top three enriched GO terms were "defense response", "immune response", and "cellular response to cytokine stimulus" in BP (Fig. 4A); and "cytokine receptor activity", "hydrolase activity", and "phospholipase activity" in MF (Fig. 4B). The results of KEGG analysis are shown in Supplementary Table S4. The top five pathways were "Cytokinecytokine receptor interaction", "Cell cycle", "Systemic lupus erythematosus", and "NODlike receptor signaling pathway" (Fig. 4C). The results of the enrichment analysis showed that most genes and pathways were associated with inflammation. Thus, a series of specific genes and pathways involved in psoriasis were closely related to inflammation, which further confirmed that psoriasis is an immune mediated inflammatory skin disease.

\section{Predication of common MREs of differentially expressed circRNAs and mRNAs}

To evaluate the potential functions of differentially expressed circRNAs in psoriasis, the putative MREs of differentially expressed circRNAs were described. A total of 4405 circRNAs were predicted to have MREs (Supplementary Table S5). Moreover, we predicated the MREs of differentially expressed mRNAs. Common MREs of differentially expressed circRNAs and mRNAs were shown in Supplementary Table S6. 


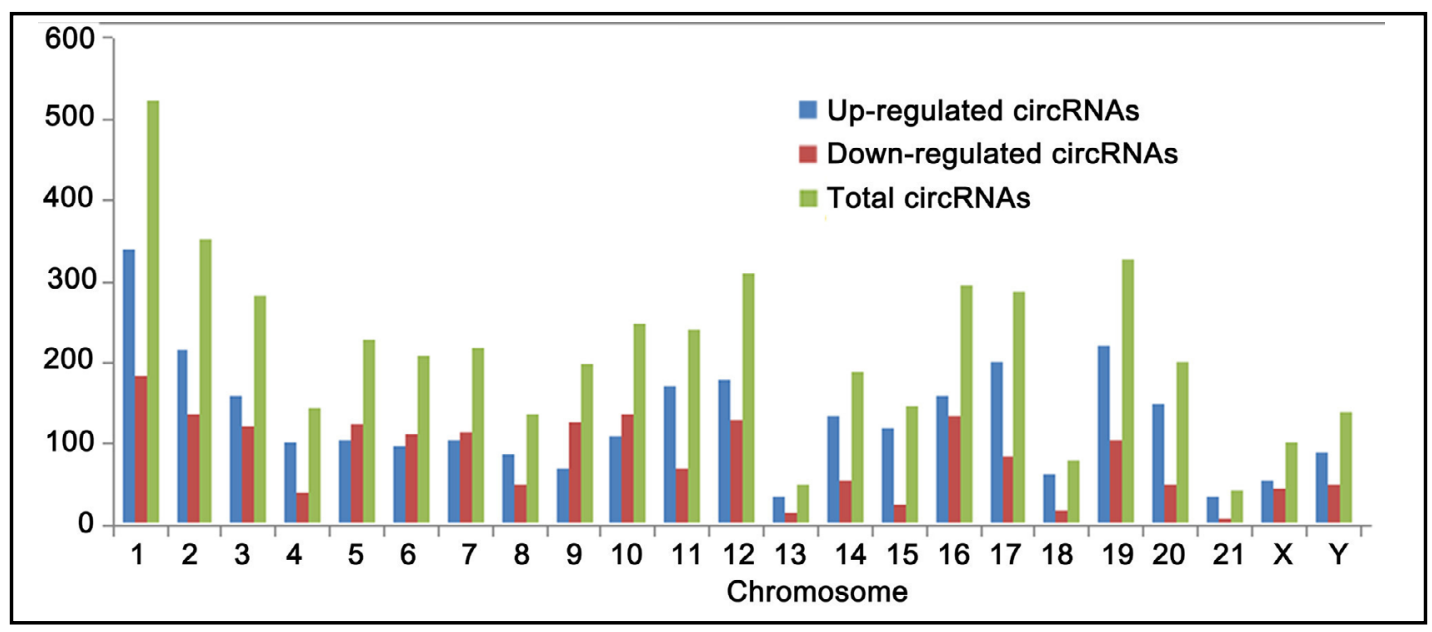

Fig. 3. Distribution of dysregulated circular RNAs (circRNAs) in human chromosomes.

\section{Validation of circRNA} expression

To verify the results from the circRNAs microarray, we selected six differentially expressed circRNAs based on the function of differentially expressed mRNAs, that have common MREs with differentially expressed circRNAs. These differentially expressed mRNAs is STAT1, FOSL1, HIF1A, and IL6 (Supplementary Table S6). All of them involves in regulating immunity and inflammation, which contributes to psoriasis [21-23]. The details of hsa_circ_0061012, hsa_circ_0069260, hsa circ_0022697,hsa_circ_0043804, hsa_circ_0031329, and hsa circ_0062533 were shown in Table 4 . The verification results using qRT-PCR analysis are shown in Fig. 5. The expression patterns of these six circRNAs from qRT-PCR results showed that only hsa_circ_0061012 expression was upregulated in psoriatic lesions compared with that in normal healthy skin tissues.
Table 3. Top 20 upregulated and downregulated (fold-change $\geq$ 2 and $\mathrm{P}<0.05$ ) circRNAs sorted by their fold change in psoriatic lesions versus normal healthy skin tissues. Note: Fold-change refers to the absolute ratio (no log scale) of normalized intensities between two groups

\begin{tabular}{|c|c|c|c|c|c|}
\hline CircRNA & P-value & Fold-change & Regulation & Chromosome & Host gene \\
\hline hsa_circ_0060531 & 0.001 & 442.297 & up & chr20 & PI3 \\
\hline hsa_circ_0060532 & 0.005 & 330.084 & up & $\operatorname{chr} 20$ & PI3 \\
\hline hsa_circ_0060533 & 0.004 & 270.233 & up & $\operatorname{chr} 20$ & PI3 \\
\hline hsa_circ_0014221 & 0.012 & 269.952 & up & chr1 & S100A9 \\
\hline hsa_circ_0014220 & 0.018 & 238.481 & up & chr1 & S100A9 \\
\hline hsa_circ_0014222 & 0.012 & 172.332 & up & chr1 & S100A8 \\
\hline hsa_circ_0082476 & 0.001 & 143.673 & up & chr7 & AKR1B10 \\
\hline hsa_circ_0024028 & 0.002 & 128.780 & up & $\operatorname{chr} 11$ & HEPHL1 \\
\hline hsa_circ_0043630 & 0.002 & 127.062 & up & $\operatorname{chr} 17$ & KRT16 \\
\hline hsa_circ_0036722 & 0.001 & 126.226 & up & $\operatorname{chr} 15$ & RHCG \\
\hline hsa_circ_0043629 & 0.001 & 123.373 & up & $\operatorname{chr} 17$ & KRT16 \\
\hline hsa_circ_0082477 & 0.001 & 113.821 & up & $\operatorname{chr} 7$ & AKR1B10 \\
\hline hsa_circ_0043627 & 0.003 & 112.113 & up & $\operatorname{chr} 17$ & KRT16 \\
\hline hsa_circ_0088732 & 0.018 & 88.896 & up & $\operatorname{chr} 9$ & LCN2 \\
\hline hsa_circ_0024027 & 0.001 & 69.580 & up & $\operatorname{chr} 11$ & HEPHL1 \\
\hline hsa_circ_0043626 & 0.004 & 69.111 & up & $\operatorname{chr} 17$ & KRT16 \\
\hline hsa_circ_0024030 & 0.000 & 68.749 & up & $\operatorname{chr} 11$ & HEPHL1 \\
\hline hsa_circ_0043625 & 0.002 & 61.240 & up & $\operatorname{chr} 17$ & KRT16 \\
\hline hsa_circ_0043628 & 0.004 & 60.214 & up & $\operatorname{chr} 17$ & KRT16 \\
\hline hsa_circ_0034646 & 0.003 & 38.131 & up & $\operatorname{chr} 15$ & CHAC1 \\
\hline hsa_circ_0009406 & 0.000 & 12.378 & down & chr1 & MEGF6 \\
\hline hsa_circ_0012485 & 0.007 & 7.531 & down & chr1 & RAB3B \\
\hline hsa_circ_0013241 & 0.021 & 7.229 & down & chr1 & F3 \\
\hline hsa_circ_0052001 & 0.001 & 7.063 & down & $\operatorname{chr} 19$ & MYH14 \\
\hline hsa_circ_0009387 & 0.012 & 6.846 & down & chr1 & PLCH2 \\
\hline hsa_circ_0062161 & 0.036 & 6.812 & down & $\operatorname{chr} 22$ & CECR2 \\
\hline hsa_circ_0061091 & 0.004 & 6.748 & down & $\operatorname{chr} 20$ & LAMA5 \\
\hline hsa_circ_0009385 & 0.003 & 6.709 & down & chr1 & PLCH2 \\
\hline hsa_circ_0013239 & 0.015 & 6.672 & down & chr1 & F3 \\
\hline hsa_circ_0070956 & 0.007 & 6.638 & down & $\operatorname{chr} 4$ & PHF17 \\
\hline hsa_circ_0071668 & 0.036 & 6.488 & down & chr5 & ZDHHC11 \\
\hline hsa_circ_0032865 & 0.012 & 6.278 & down & $\operatorname{chr} 14$ & EML5 \\
\hline hsa_circ_0087523 & 0.000 & 6.223 & down & chr9 & WNK2 \\
\hline hsa_circ_0009383 & 0.009 & 6.185 & down & chr1 & PLCH2 \\
\hline hsa_circ_0001946 & 0.001 & 6.128 & down & $\operatorname{chrX}$ & CDR1 \\
\hline hsa_circ_0089755 & 0.005 & 6.087 & down & $\operatorname{chr} 9$ & EHMT1 \\
\hline hsa_circ_0072455 & 0.003 & 5.983 & down & $\operatorname{chr} 5$ & FST \\
\hline hsa_circ_0069182 & 0.008 & 5.854 & down & $\operatorname{chr} 4$ & SLC2A9 \\
\hline hsa_circ_0049170 & 0.002 & 5.737 & down & $\operatorname{chr} 19$ & OLFM2 \\
\hline hsa_circ_0030018 & 0.047 & 5.429 & down & $\operatorname{chr} 13$ & POSTN \\
\hline
\end{tabular}




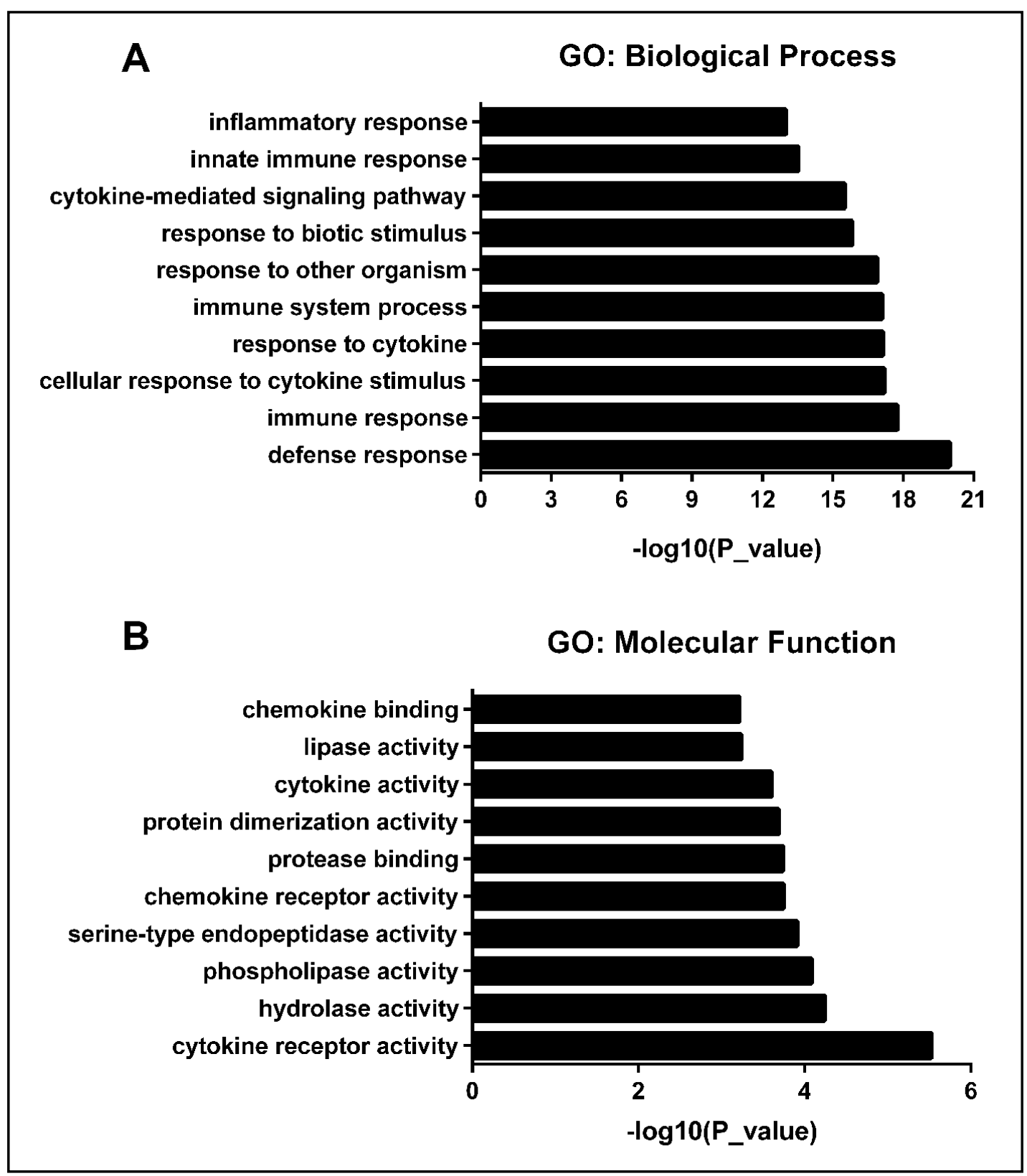

Fig. 4. Gene ontology (GO) and Kyoto Encyclopedia of Genes and Genomes (KEGG) analysis. A: Top 10 GO enriched biological process terms for the differentially expressed mRNAs. B: Top 10 GO enriched molecular function terms for differentially expressed mRNAs. C: Top 10 KEGG pathways enriched for differentially expressed mRNAs.

Table 4. Detail of six circRNAs that were chosen for qRT-PCR validation. Note: Fold-change refers to the absolute ratio (no log scale) of normalized intensities between two groups

\begin{tabular}{lcccccc}
\hline CircRNA & P-value & Fold-change & Regulation & Chromosome & Sequence length & Host gene \\
\hline hsa_circ_0061012 & 0.003 & 3.059 & up & chr20 & 446 & SLMO2-ATP5E \\
hsa_circ_0069260 & 0.000 & 3.108 & up & chr4 & 1399 & LAP3 \\
hsa_circ_0022697 & 0.000 & 2.188 & up & chr11 & 2927 & RPS6KA4 \\
hsa_circ_0043804 & 0.003 & 2.750 & up & chr17 & 156 & STAT3 \\
hsa_circ_0031329 & 0.000 & 4.021 & up & chr14 & 502 & PSME2 \\
hsa_circ_0062533 & 0.021 & 2.168 & up & chr13 & 519 & DIAPH3 \\
\hline
\end{tabular}


Fig. 5. Expression levels of hsa circ_0061012, hsa_circ_0069260, hsa_ circ_0022697, hsa_circ_0043804, hsa_ circ_0031329, and hsa_circ_0062533 in psoriatic lesions compared with that in normal healthy skin tissues. The results of quantitative real-time reverse transcription PCR (qRTPCR) were evaluated using the $2^{-\Delta \Delta C T}$ method. Results are represented as means \pm standard deviation (SD). ${ }^{*} \mathrm{P}$ values $<0.05$.

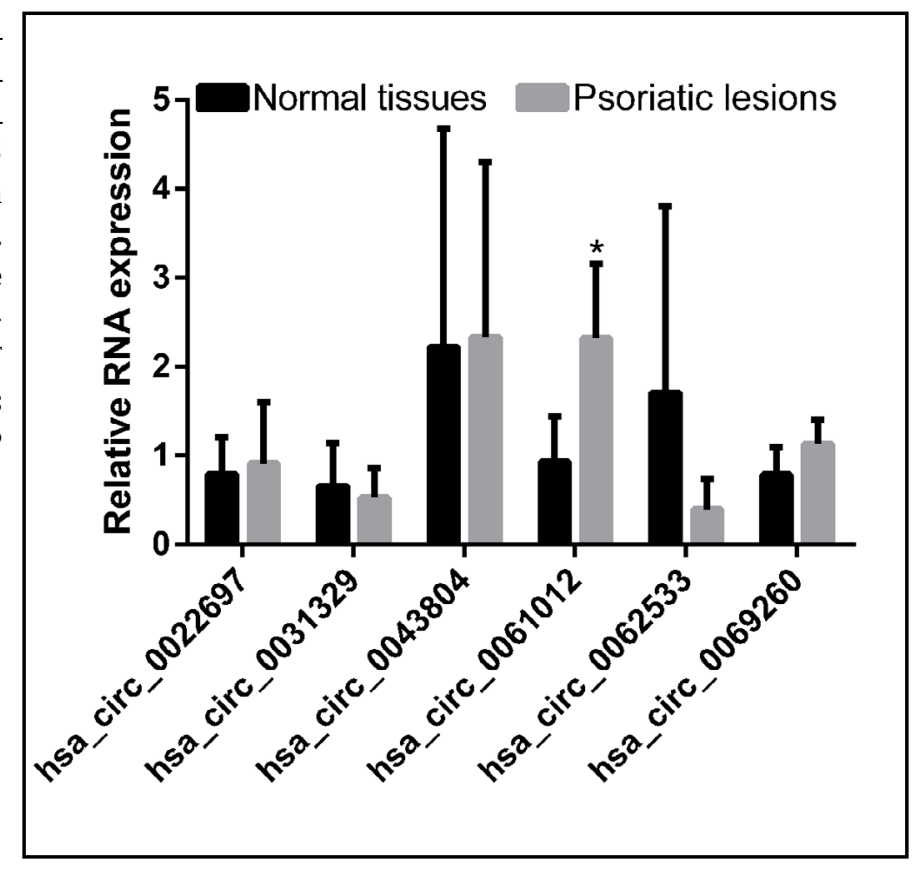

Fig. 6. Circular RNA (circRNA)/ microRNA (miRNA) interaction network for hsa_circ_0061012. The interaction network of differentially expressed circRNAs (red nodes) and their five complementary binding miRNAs (blue nodes) was presented by the cytoscape software.

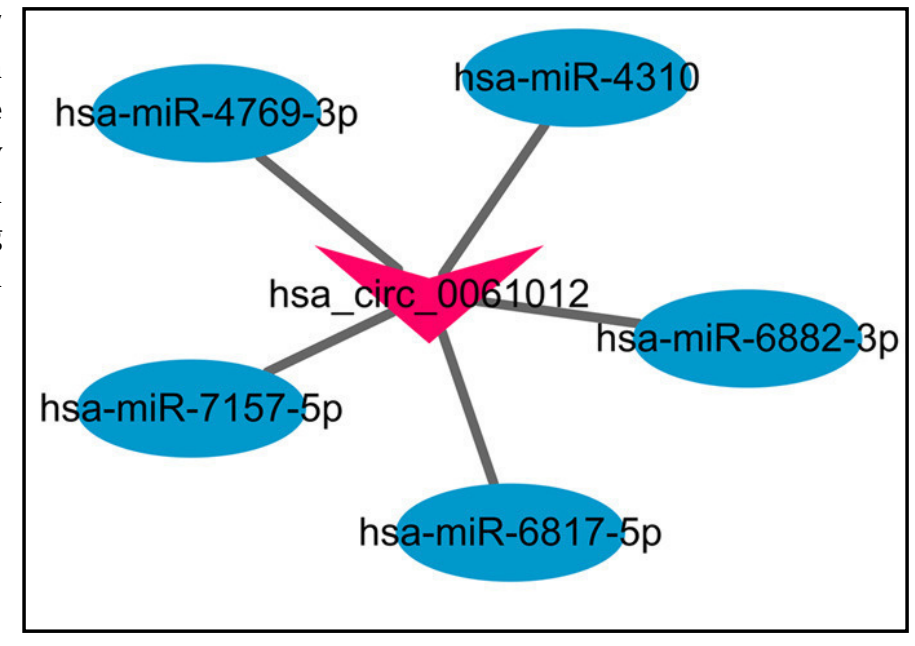

MiRNA response elements analysis of differentially expressed hsa_circ_0061012

To further investigate the regulatory mechanism of hsa_circ_0061012, we analyzed MREs in hsa_circ_0061012 using the Arraystar miRNA target prediction software. The results showed that the top five MREs of hsa_circ_0061012 are hsa-miR-7157-5p, hsa-miR4769-3p, hsa-miR-6817-5p, hsa-miR-4310, and hsa-miR-6882-3p (Fig. 6). Following, we predicated the targets of these five miRNAs. The targets that were upregulated in psoriatic lesions were used to construct a miRNA and target network, shown in Supplementary Fig. S1. Most targets of hsa-miR-7157-5p and hsa-miR-4310 are overlapped. The target number of hsa-miR-4769-3p is most and the target number of hsa-miR-6817-5p is least.

GO analysis of the targets of five miRNAs

To further investigate the regulatory mechanism of hsa_circ_0061012, G0 analysis of the targets of five miRNAs were carried out. As shown in Fig. 7, most of top 30 of GO enrichment are related to psoriasis, such as T cell selection [24], response to interleukin 4 [25], and regulation of NF-kappaB import into nucleus [26]. 


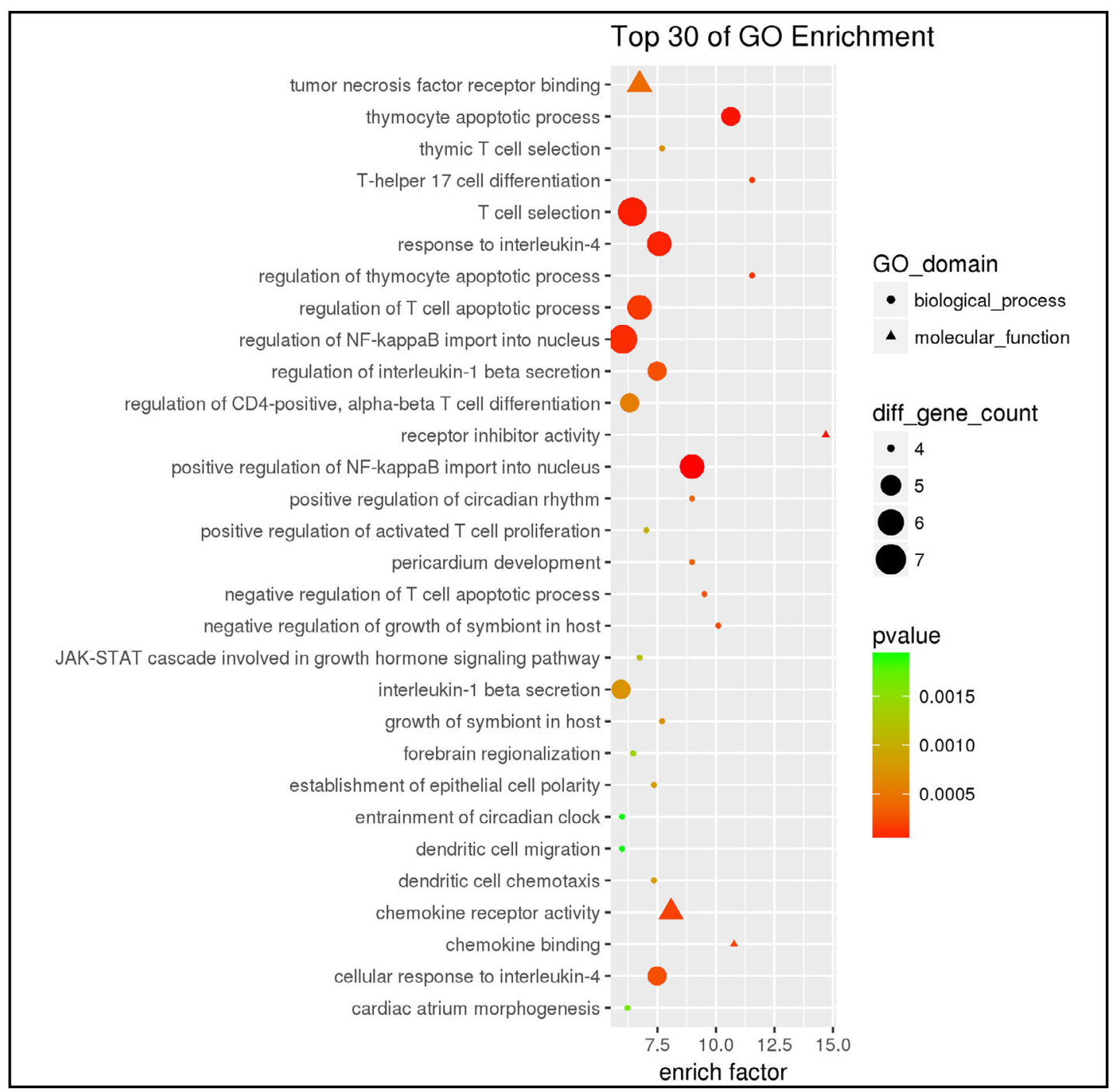

Fig. 7. GO analysis of the upregulated targets in psoriatic lesions of five miRNAs.

\section{Discussion}

Psoriatic skin is characterized by inflammation infiltration of the dermis and hyper proliferation of the epidermis [27]. Although the etiology of psoriasis remains unknown, it is a complex, multifactorial disorder that is influenced by genetic, epigenetic, and environmental factors [27]. T cell- and immune active molecules-mediated inflammatory reactions play an important role. To date, different systematic drugs and biologic therapies have been used to treat moderate to severe psoriasis [28]. However, these treatments are not curative, and the study of the pathogenesis of psoriasis is ongoing. circRNAs have been reported to play essential roles in regulating immunity, inflammation, and cell proliferation [18-20]. It is known that psoriasis is an immune-mediated, chronic inflammatory disorder that is mediated by elements of the innate and adaptive immune systems [29-31]. So circRNAs may be an important regulatory factor in the development of psoriasis and may be new therapeutic target for psoriasis. However, the expression profile of circRNAs and their potential biological functions in psoriasis have not been reported so far. In the present study, we utilized circRNA microarray to provide comprehensive circRNA expression profile between psoriatic lesions and normal healthy skin tissues, in an attempt to explore the 


\section{Cellular Physiology Cell Physiol Biochem 2018;50:15-27 \begin{tabular}{l|l} 
DOI: 10.1159/000493952 & $\begin{array}{l}\text { O 2018 The Author(s). Published by S. Karger AG, Basel } \\
\text { www.karger.com/cpb }\end{array}$
\end{tabular} \\ Qiao et al.: Circular RNA Expression Profile in Psoriasis}

possible involvement of dysregulated circRNAs in the development of psoriasis. We identified a large number of differentially expressed circRNAs from diverse genomic locations: 3016 circRNAs were significantly upregulated and 1940 were downregulated between two groups. This is the first report about the comprehensive circRNA expression profile in psoriasis and our results will give a novel theoretical basis for further studies on the function of circRNA in psoriasis.

circRNAs are rich in miRNA binding sites (miRNA response elements, MREs), and they are believed to playing a role of miRNAs sponges in cells, leading to relief of the inhibitory effect of miRNAs on their target genes and, ultimately, increased expression of the target genes. In this mechanism the circRNAs act as competitive endogenous RNAs (ceRNAs) [15, 32]. CircRNAs play an important role in the regulation of diseases via their interaction with disease-associated miRNAs [33]. Our results showed that all 4405 dysregulated circRNAs had miRNAs binding sites, and were thus predicted to play a regulatory role via the ceRNA mechanism.

In this study, we used qRT-PCR validation for six circRNAs in the 10 psoriatic samples. hsa_circ_0061012 was significantly upregulated (fold change 2.49) between the two groups, and the difference was statistically significant $(\mathrm{P}<0.05)$. hsa_circ_0061012 has five miRNA binding sites (hsa-miR-7157-5p, hsa-miR-4769-3p, hsa-miR-6817-5p, hsa-miR-4310, and hsa-miR-6882-3p). To further investigate the potential regulatory role of hsa_circ_0061012 in psoriasis, we predicated the targets of these five miRNAs, and GO analysis was carried out to investigate the biological functions enriched among the upregulated targets in psoriasis. The $\mathrm{GO}$ analysis identified that most of top 30 of GO enrichment are related to psoriasis, such as $\mathrm{T}$ cell selection [24], response to interleukin 4 [25], and regulation of NF-kappaB import into nucleus [26]. These results suggested that aberrant expression of hsa_circ_0061012 might be involved in the pathogenesis of psoriasis. However, the detailed regulating mechanism needs further study.

In a future study, we will screen more differentially expressed circRNAs and expand the sample size of verification. This is a limitation of this paper, and it is also important for further research. hsa_circ_0061012 knockdown or overexpression experiments will be used to further evaluate the potential role of hsa_circ_0061012 functioned as ceRNA in psoriasis.

\section{Conclusion}

In conclusion, this is the first report of using a microarray to investigate the circRNA expression profile in psoriasis. hsa_circ_0061012 was significantly upregulated in psoriatic lesions, indicating that hsa_circ_0061012 might be a candidate biomarker for psoriasis. Taken together, our research results provide a new perspective toward a better understanding of ceRNA-mediated gene regulation in psoriasis, and provide a novel theoretical basis for further studies on the function of circRNA in psoriasis.

\section{Acknowledgements}

This work was supported by the National Natural Science Foundation of China (No. 81573046) and the Key Project of Science and Technology of Shandong province (No. 2014GSF118125).

\section{Disclosure Statement}

The authors declare that they have no conflict of interests. 


\section{Cellular Physiology Cell Physiol Biochem 2018;50:15-27

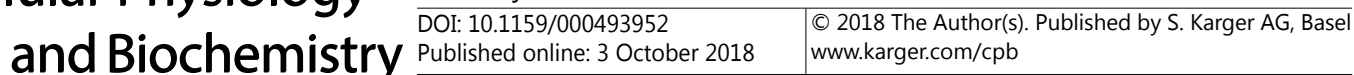 \\ Qiao et al.: Circular RNA Expression Profile in Psoriasis}

\section{References}

1 Cai Y, Fleming C, Yan J: New insights of T cells in the pathogenesis of psoriasis. Cell Mol Immunol 2012;9:302-309.

-2 Chandran V, Raychaudhuri SP: Geoepidemiology and environmental factors of psoriasis and psoriatic arthritis. J Autoimmun 2010;34:J314-321.

-3 Parisi R, Symmons DP, Griffiths CE, Ashcroft DM: Global epidemiology of psoriasis: a systematic review of incidence and prevalence. J Invest Dermatol 2013;133:377-385.

-4 Nguyen CM, Beroukhim K, Danesh MJ, Babikian A, Koo J, Leon A: The psychosocial impact of acne, vitiligo, and psoriasis: a review. Clin Cosmet Investig Dermatol 2016;9:383-392.

5 Lowes MA, Russell CB, Martin DA, Towne JE, Krueger JG: The IL-23/T17 pathogenic axis in psoriasis is amplified by keratinocyte responses. Trends Immunol 2013;34:174-181.

6 Schaarschmidt ML, Kromer C, Herr R, Schmieder A, Goerdt S, Peitsch WK: Treatment satisfaction of patients with psoriasis. Acta Derm Venereol 2015;95:572-578.

7 Zibert JR, Lovendorf MB, Litman T, Olsen J, Kaczkowski B, Skov L: MicroRNAs and potential target interactions in psoriasis. J Dermatol Sci 2010;58:177-185.

8 Gupta R, Ahn R, Lai K, Mullins E, Debbaneh M, Dimon M, Arron S, Liao W: Landscape of Long Noncoding RNAs in Psoriatic and Healthy Skin. J Invest Dermatol 2016;136:603-609.

-9 Wilusz JE: Circular RNAs: Unexpected outputs of many protein-coding genes. RNA Biol 2017;14:10071017.

10 Vicens Q, Westhof E: Biogenesis of Circular RNAs. Cell 2014;159:13-14.

11 Cocquerelle C, Mascrez B, Hetuin D, Bailleul B: Mis-splicing yields circular RNA molecules. Faseb J 1993;7:155-160.

$>12$ Suzuki H, Zuo Y, Wang J, Zhang MQ Malhotra A, Mayeda A: Characterization of RNase R-digested cellular RNA source that consists of lariat and circular RNAs from pre-mRNA splicing. Nucleic Acids Res 2006;34:e63.

13 Han B, Chao J, Yao H: Circular RNA and its mechanisms in disease: From the bench to the clinic. Pharmacol Ther 2018;187:31-44.

14 Greene J, Baird AM, Brady L, Lim M, Gray SG, McDermott R, Finn SP: Circular RNAs: Biogenesis, Function and Role in Human Diseases. Front Mol Biosci 2017;4:38.

15 Hansen TB, Jensen TI, Clausen BH, Bramsen JB, Finsen B, Damgaard CK, Kjems J: Natural RNA circles function as efficient microRNA sponges. Nature 2013;495:384-388.

16 Yuan Y, Liu W, Zhang Y, Sun S: CircRNA circ_0026344 as a prognostic biomarker suppresses colorectal cancer progression via microRNA-21 and microRNA-31. Biochem Biophys Res Commun 2018;23:3140131403.

17 Qu S, Zhong Y, Shang R, Zhang X, Song W, Kjems J, Li H: The emerging landscape of circular RNA in life processes. RNA Biol 2017;14:992-999.

18 Zhou Z, Du D, Chen A, Zhu L: Circular RNA expression profile of articular chondrocytes in an IL-1betainduced mouse model of osteoarthritis. Gene 2018;644:20-26.

19 Li CY, Ma L, Yu B: Circular RNA hsa_circ_0003575 regulates oxLDL induced vascular endothelial cells proliferation and angiogenesis. Biomed Pharmacother 2017;95:1514-1519.

20 Wang M, Yu F, Wu W, Zhang Y, Chang W, Ponnusamy M, Wang K, Li P: Circular RNAs: A novel type of noncoding RNA and their potential implications in antiviral immunity. Int J Biol Sci 2017;13:1497-1506.

-21 Bai L, Fang H, Xia S, Zhang R, Li L, Ochando J, Xu J, Ding Y: STAT1 activation represses IL-22 gene expression and psoriasis pathogenesis. Biochem Biophys Res Commun 2018;501:563-569.

22 Sobolev VV, Zolotorenko AD, Soboleva AG, Elkin AM, Il'ina SA, Serov DN, Potekaev NN, Tkachenko SB, Minnibaev MT, Piruzyan AL: Effects of expression of transcriptional factor AP-1 FOSL1 gene on psoriatic process. Bull Exp Biol Med 2011;150:632-634.

23 Torales-Cardena A, Martinez-Torres I, Rodriguez-Martinez S, Gomez-Chavez F, Cancino-Diaz JC, VazquezSanchez EA, Cancino-Diaz ME: Cross Talk between Proliferative, Angiogenic, and Cellular Mechanisms Orchestred by HIF-1alpha in Psoriasis. Mediators Inflamm 2015;607363:7.

-24 Sobell JM, Kalb RE, Weinberg JM: Management of moderate to severe plaque psoriasis (part 2): clinical update on T-cell modulators and investigational agents. J Drugs Dermatol 2009;8:230-238. 


\section{Cellular Physiology Cell Physiol Biochem 2018;50:15-27 \begin{tabular}{l|l} 
DOI: 10.1159/000493952 & $\begin{array}{l}\text { O 2018 The Author(s). Published by S. Karger AG, Basel } \\
\text { www.karger.com/cpb }\end{array}$ \\
\cline { 2 - 3 }
\end{tabular} \\ Qiao et al.: Circular RNA Expression Profile in Psoriasis}

25 Chen ZW, Zhang YB, Chen XJ, Liu X, Wang Z, Zhou XK, Qiu J, Zhang NN, Teng X, Mao YQ Liu CY, Wei YQ Li J: Retinoic Acid promotes interleukin-4 plasmid-dimethylsulfoxide topical transdermal delivery for treatment of psoriasis. Ann Dermatol 2015;27:121-127.

26 Dou R, Liu Z, Yuan X, Xiangfei D, Bai R, Bi Z, Yang P, Yang Y, Dong Y, Su W, Li D, Mao C: PAMs ameliorates the imiquimod-induced psoriasis-like skin disease in mice by inhibition of translocation of NF-kappaB and production of inflammatory cytokines. 2017;12:e0176823.

27 Mahajan R, Handa S: Pathophysiology of psoriasis. Indian J Dermatol Venereol Leprol 2013;79:S1-9.

-28 Ovejero-Benito MC, Munoz-Aceituno E, Reolid A, Saiz-Rodriguez M, Abad-Santos F: Pharmacogenetics and Pharmacogenomics in Moderate-to-Severe Psoriasis. Am J Clin Dermatol 2017;19:209-222.

29 Buchau AS, Gallo RL: Innate immunity and antimicrobial defense systems in psoriasis. Clin Dermatol 2007;25:616-624.

30 Bos JD, de Rie MA, Teunissen MB, Piskin G: Psoriasis: dysregulation of innate immunity. Br J Dermatol 2005;152:1098-1107.

-31 Sigurdardottir SL, Thorleifsdottir RH, Valdimarsson H, Johnston A: The role of the palatine tonsils in the pathogenesis and treatment of psoriasis. Br J Dermatol 2013;168:237-242.

-32 Qu S, Yang X, Li X, Wang J, Gao Y, Shang R, Sun W, Dou K, Li H: Circular RNA: A new star of noncoding RNAs. Cancer Lett 2015;365:141-148.

-33 Haque S, Harries LW: Circular RNAs (circRNAs) in Health and Disease. Genes 2017;8:353. 